\title{
Proof of a conjecture of Kløve on permutation codes under the Chebychev distance
}

\author{
Victor J. W. Guo ${ }^{1}$ - Yiting Yang $^{2}$
}

Received: 16 October 2015 / Revised: 30 June 2016 / Accepted: 9 July 2016 /

Published online: 4 August 2016

(C) The Author(s) 2016. This article is published with open access at Springerlink.com

Abstract Let $d$ be a positive integer and $x$ a real number. Let $A_{d, x}$ be a $d \times 2 d$ matrix with its entries

$$
a_{i, j}= \begin{cases}x & \text { for } 1 \leqslant j \leqslant d+1-i \\ 1 & \text { for } d+2-i \leqslant j \leqslant d+i \\ 0 & \text { for } d+1+i \leqslant j \leqslant 2 d\end{cases}
$$

Further, let $R_{d}$ be a set of sequences of integers as follows:

$$
R_{d}=\left\{\left(\rho_{1}, \rho_{2}, \ldots, \rho_{d}\right) \mid 1 \leqslant \rho_{i} \leqslant d+i, 1 \leqslant i \leqslant d, \text { and } \rho_{r} \neq \rho_{s} \text { for } r \neq s\right\} .
$$

and define

$$
\Omega_{d}(x)=\sum_{\rho \in R_{d}} a_{1, \rho_{1}} a_{2, \rho_{2}} \ldots a_{d, \rho_{d}} .
$$

In order to give a better bound on the size of spheres of permutation codes under the Chebychev distance, Kløve introduced the above function and conjectured that

$$
\Omega_{d}(x)=\sum_{m=0}^{d}\left(\begin{array}{l}
d \\
m
\end{array}\right)(m+1)^{d}(x-1)^{d-m} .
$$

In this paper, we settle down this conjecture positively.

Communicated by D. Jungnickel.

\section{Yiting Yang}

ytyang@tongji.edu.cn

Victor J. W. Guo

jwguo@hytc.edu.cn

1 School of Mathematical Sciences, Huaiyin Normal University, Huai'an 223300, Jiangsu, People's Republic of China

2 Department of Mathematics, Tongji University, Shanghai 200092, People's Republic of China 
Keywords Permutation code $\cdot$ Chebychev distance $\cdot$ Permanent

Mathematics Subject Classification 05A05 - 94B65

\section{Introduction}

A permutation code is a subset of the symmetric group $S_{n}$, equipped with a distance metric. Permutation codes are of potential use in various applications such as power-line communications and coding for flash memories used with rank modulation [6,7]. Permutation codes were extensively studied over the last decade. Hamming metric is naturally the first to be considered. Later, Ulam metric [4] and Kendall $\tau$-metric [2] were introduced and are now the two most investigated metrics. However in [9], a new metric named the Chebyshev metric was proposed by Kløve et al., when they were studying the multi-level flash memory model. A combinatorial survey on metrics related to permutations was given in [3].

The two main questions in coding theory are fundamental limits on the parameters of the code (information rate versus minimum distance) and constructions of codes that attain these limits. It turns out that both topics are difficult for permutation codes. Few explicit constructions were obtained for various metrics and no general bounds better than the GV-bound and Sphere packing bounds were found in [1,2,4,6,9] except for the Hamming metric [5]. Both the GV-bound and the Sphere packing bound depends on the volume $(V(d, n))$ of a typical "ball" which consists of permutations in $S_{n}$ at distance at most $d$ from the identity permutation. The calculation of the volume of that ball becomes a crucial problem.

The Chebychev distance $d(p, q)$ between two permutations $p=\left(p_{1}, p_{2}, \ldots, p_{n}\right)$ and $q=\left(q_{1}, q_{2}, \ldots, q_{n}\right)$ is defined by

$$
d(p, q)=\max _{j}\left|p_{j}-q_{j}\right|
$$

Let

$$
T_{d, n}=\left\{p \in S_{n} \| p_{i}-i \mid \leqslant d \quad \text { for } 1 \leqslant i \leqslant n\right\} .
$$

It is clear that $V(d, n)=\left|T_{d, n}\right|$. The permanent of an $n \times n$ matrix $A$ is defined by

$$
\operatorname{per} A=\sum_{p \in S_{n}} a_{1, p_{1}} \ldots a_{n, p_{n}} .
$$

Let $A^{(d, n)}$ be the $n \times n$ matrix with $a_{i, j}^{d, n}=1$ if $|i-j| \leqslant d$ and $a_{i, j}^{d, n}=0$ otherwise. Clearly, $V(d, n)=\operatorname{per} A^{(d, n)}$. Although the permanent looks similar to the determinant of a matrix, it is a difficult problem to compute the permanent for general matrices. The celebrated van der Waerden theorem gives a lower bound for the permanent of the so called doubly stochastic $n \times n$ matrix. Here doubly stochastic means that all the elements are non-negative and that the sum of the elements in any row or column is 1 . Thus, if $A$ is an $n \times n$ matrix where the sum of the elements in any row or column is a constant $k$, then van der Waerden theorem gives a lower bound on the permanent of $A$.

By noticing that most rows and columns of $A^{(d, n)}$ have the sum $2 d+1$, Kløve defined a closely related matrix $B^{(d, n)}$ with row sum and column sum $2 d+1$ so that van der Waerden's theorem can be applied. The matrix $B^{(d, n)}$ is defined as follows: 


$$
b_{i, j}= \begin{cases}0 & \text { if } i>j+d \text { or } j>i+d \\ 2 & \text { if } i+j \leqslant d+1 \text { or } i+j \geqslant 2 n+1-d, \\ 1 & \text { otherwise. }\end{cases}
$$

With this new defined matrix $B^{(d, n)}$, Kløve [10] gave a lower bound on $V(d, n)$ as follows:

$$
V(d, n)>\frac{\sqrt{2 \pi n}}{2^{2 d}}\left(\frac{2 d+1}{e}\right)^{n} .
$$

Let $A_{d, 2}=\left(a_{i, j}\right)$ be the upper left corner of $B^{(d, n)}$ which is a $d \times 2 d$ matrix defined by

$$
a_{i, j}= \begin{cases}2, & \text { if } 1 \leqslant j \leqslant d+1-i \\ 1, & \text { if } d+2-i \leqslant j \leqslant d+i \\ 0, & \text { if } d+i+1 \leqslant j \leqslant 2 d\end{cases}
$$

For example,

$$
A_{1,2}=\left(\begin{array}{ll}
2 & 1
\end{array}\right), \quad A_{2,2}=\left(\begin{array}{llll}
2 & 2 & 1 & 0 \\
2 & 1 & 1 & 1
\end{array}\right), \quad A_{3,2}=\left(\begin{array}{llllll}
2 & 2 & 2 & 1 & 0 & 0 \\
2 & 2 & 1 & 1 & 1 & 0 \\
2 & 1 & 1 & 1 & 1 & 1
\end{array}\right) .
$$

Let $R_{d}$ be a set of sequences of integers as follows:

$$
R_{d}=\left\{\left(\rho_{1}, \rho_{2}, \ldots, \rho_{d}\right) \mid 1 \leqslant \rho_{i} \leqslant d+i, 1 \leqslant i \leqslant d \text {, and } \rho_{r} \neq \rho_{s}\right\} .
$$

Define

$$
\Omega_{d}=\sum_{\rho \in R_{d}} a_{1, \rho_{1}} a_{2, \rho_{2}} \ldots a_{d, \rho_{d}}
$$

Let

$$
\omega_{d}=\frac{\Omega_{d} e^{d}}{(2 d+1)^{d}} .
$$

Kløve [9] also gave the following lower bound on $V(d, n)$ :

$$
V(d, n)>\frac{\sqrt{2 \pi(n+2 d)}}{\omega_{d}^{2}}\left(\frac{2 d+1}{e}\right)^{n} .
$$

Thus whether (1.2) is an improvement compared with (1.1) depends on the value $\Omega_{d}$. Kløve [10] gave the first 9 values of $\Omega_{d}$ as follows:

3, 18, 170, 2200, 36232, 725200, 17095248, 463936896, 14246942336,

which coincide the sequence A074932 in [12], and made the following conjecture.

Conjecture 1 [10, Conjecture 1] For any positive integer $d$,

$$
\Omega_{d}=\sum_{m=0}^{d}\left(\begin{array}{l}
d \\
m
\end{array}\right)(m+1)^{d}
$$

Kløve showed that the equation (1.2) improves on (1.1) if Conjecture 1 is true. Furthermore, let $A_{d, x}=\left(a_{i, j}\right)$ be the $d \times 2 d$ matrix defined by

$$
a_{i, j}=\left\{\begin{array}{lll}
x, & \text { if } \quad 1 \leqslant j \leqslant d+1-i \\
1, & \text { if } \quad d+2-i \leqslant j \leqslant d+i \\
0, & \text { if } \quad d+i+1 \leqslant j \leqslant 2 d
\end{array}\right.
$$


and let

$$
\Omega_{d}(x)=\sum_{\rho \in R_{d}} a_{1, \rho_{1}} a_{2, \rho_{2}} \ldots a_{d, \rho_{d}} .
$$

In particular, $\Omega_{d}(2)=\Omega_{d}$. Kløve gave the following generalized conjecture and verified it for $d \leqslant 9$.

Conjecture 2 [10, Conjecture 1] For any positive integer $d$,

$$
\Omega_{d}(x)=\sum_{m=0}^{d}\left(\begin{array}{l}
d \\
m
\end{array}\right)(m+1)^{d}(x-1)^{d-m} .
$$

In this paper, we shall prove that Conjecture 2 is true.

\section{Proof of Kløve's Conjecture}

Theorem 3 For any positive integer d, the identity (1.3) holds.

Actually, for any $m \times n$ matrix $A=\left(a_{i, j}\right)$ with $m \leqslant n$, the permanent function of $A$ is already defined as follows (see, for example, [11]):

$$
\operatorname{per}(A)=\sum_{\sigma \in P(n, m)} a_{1, \sigma_{1}} a_{2, \sigma_{2}} \cdots a_{m, \sigma_{m}}
$$

where $P(n, m)$ denotes the set of all $m$-permutations of the $n$-set $\{1,2, \ldots, n\}$.

In fact, by the definition of $R_{d}$, we know that $R_{d}$ is exactly the subset of all $d$-permutations of the $2 d$-set $\{1,2, \ldots, 2 d\}$ such that $\sigma \in R_{d}$ if and only if $a_{1, \sigma_{1}} a_{2, \sigma_{2}} \cdots a_{d, \sigma_{d}} \neq 0$. Hence we have $\Omega_{d}(x)=\operatorname{per}\left(A_{d, x}\right)$.

In order to prove Theorem 3 , we first give a related combinatorial identity.

Lemma 4 Let $m$ and $n$ be positive integers. Then

$$
\sum_{1 \leqslant k_{1} \leqslant k_{2} \leqslant \cdots \leqslant k_{m} \leqslant n} \prod_{i=0}^{m} k_{i}(n+m-i)^{k_{i+1}-k_{i}}=\left(\begin{array}{c}
n+m-1 \\
m
\end{array}\right) n^{n+m-1},
$$

where $k_{0}=1$ and $k_{m+1}=n$.

For example, we have

$$
\sum_{1 \leqslant k_{1} \leqslant k_{2} \leqslant k_{3} \leqslant n} k_{1} k_{2} k_{3}(n+3)^{k_{1}-1}(n+2)^{k_{2}-k_{1}}(n+1)^{k_{3}-k_{2}} n^{n-k_{3}}=\left(\begin{array}{c}
n+2 \\
3
\end{array}\right) n^{n+2} .
$$


Proof of Lemma 4 We compute the multiple sum in the order from $k_{m}$ to $k_{1}$. It can be proved by induction on $k_{m-1}, k_{m-2}, \ldots, k_{m-i-1}$ respectively that

$$
\begin{aligned}
& \sum_{k_{m}=k_{m-1}}^{n} k_{m}(n+1)^{k_{m}-k_{m-1}} n^{n-k_{m}}=\left(n-k_{m-1}+1\right) n^{n-k_{m-1}+1}, \\
& \quad \sum_{k_{m-1}=k_{m-2}}^{n} k_{m-1}\left(n-k_{m-1}+1\right)(n+2)^{k_{m-1}-k_{m-2}} n^{n-k_{m-1}+1}=\left(\begin{array}{c}
n-k_{m-2}+2 \\
2
\end{array}\right) n^{n-k_{m-2}+2}, \\
& \ldots, \\
& \sum_{k_{m-i}=k_{m-i-1}}^{n} k_{m-i}\left(\begin{array}{c}
n-k_{m-i}+i \\
i
\end{array}\right)(n+i+1)^{k_{m-i}-k_{m-i-1} n^{n-k_{m-i}+i}} \\
& =\left(\begin{array}{c}
n-k_{m-i-1}+i+1 \\
i+1
\end{array}\right) n^{n-k_{m-i-1}+i+1} .
\end{aligned}
$$

By choosing $i=m-1$ in (2.2), we complete the proof of (2.1).

Proof of Theorem 3 It is clear that (1.3) is equivalent to

$$
\Omega_{d}(x+1)=\sum_{m=0}^{d}\left(\begin{array}{l}
d \\
m
\end{array}\right)(d-m+1)^{d} x^{m} .
$$

Therefore, it suffices to show that the coefficient $b_{m}$ of $x^{m}$ in $\Omega_{d}(x+1)$ is equal to $\left(\begin{array}{l}d \\ m\end{array}\right)(d-$ $m+1)^{d}$. By the definition of $\Omega_{d}(x+1)$, we know that each $x$ comes from the first term in $x+1$.

To compute $b_{m}$, we first choose $m x$ 's from $m(x+1)$ 's which are not in the same row nor in the same column of the matrix $A_{d, x+1}$, and then choose $(d-m) 1$ 's in the other $d-m$ rows so that no 1's are in the same column. Suppose that the $m x$ 's are chosen from the rows indexed by $d+1-i_{1}, d+1-i_{2}, \ldots, d+1-i_{m}$ with $i_{1}<i_{2}<\cdots<i_{m}$, respectively. By noticing that the $(d+1-i)$-th row has $i(x+1)$ 's and all the $x$ 's we choose must be in different columns, we have $i_{1}\left(i_{2}-1\right)\left(i_{3}-2\right) \cdots\left(i_{m}-m+1\right)$ ways to do this. As for the number of ways to choose 1's in the remaining rows, we notice that the $i$-th row has $d+i$ 's including those 1's in $(x+1)$ 's and all these 1's form several right trapezoids in the matrix $A_{d, x+1}$. Therefore, there are $(d+1)^{i_{1}-1} d^{i_{2}-i_{1}-1}(d-1)^{i_{3}-i_{2}-1} \cdots(d-m+1)^{d-i_{m}}$ ways to choose the remaining 1 's. It follows that

$$
\begin{aligned}
b_{m}= & \sum_{\substack{1 \leqslant i_{1}<i_{2}<\cdots<i_{m} \leqslant d\\
}} i_{1}\left(i_{2}-1\right)\left(i_{3}-2\right) \cdots\left(i_{m}-m+1\right) \\
= & \sum_{1 \leqslant k_{1} \leqslant k_{2} \leqslant \cdots \leqslant k_{m} \leqslant d-m+1} \prod_{i=0}^{m} k_{i}(d+1-i)^{k_{1}-1} d^{i_{2}-i_{1}-1}\left(d-k_{i}\right.
\end{aligned}
$$

where $k_{s}=i_{s}-s+1(s=1, \ldots, m), k_{0}=1$, and $k_{m+1}=d-m+1$. By replacing $n$ by $d-m+1$ in (2.1), we obtain $b_{m}=\left(\begin{array}{l}d \\ m\end{array}\right)(d-m+1)^{d}$. This completes the proof.

Acknowledgements The authors thank the anonymous referees for their helpful comments on a previous version of this paper. The first author was partially supported by the National Natural Science Foundation of China under Grant No. 11371144 and the Qing Lan Project of Jiangsu Province. The second author was partially supported by the National Natural Science Foundation of China under Grant No. 11101360 and Outstanding Young Scholar Foundation of Tongji University under Grant No. 2013KJ031. 
Open Access This article is distributed under the terms of the Creative Commons Attribution 4.0 International License (http://creativecommons.org/licenses/by/4.0/), which permits unrestricted use, distribution, and reproduction in any medium, provided you give appropriate credit to the original author(s) and the source, provide a link to the Creative Commons license, and indicate if changes were made.

\section{References}

1. Barg A., Mazumdar A.: Codes in permutations and error correction for rank modulation. IEEE Trans. Inf. Theory 56(7), 3158-3165 (2010).

2. Buzaglo S., Etzion T.: Bounds on the size of permutation codes with the Kendall $\tau$-metric. IEEE Trans. Inf. Theory 61(6), 3241-3249 (2015).

3. Deza M., Huang H.: Metrics on permutations, a survey. J. Comb. Inf. Syst. Sci. 23, 173-185 (1998).

4. Farnoud F., Skachek V., Milenkovic O.: Error-corection in flash memories via codes in the Ulam metric. IEEE Trans. Inf. Theory 59(5), 3003-3020 (2013).

5. Gao F., Yang Y., Ge G.: An improvement to Gilbert-Varshamov bound for permutation codes. IEEE Trans. Inf. Theory 50, 1655-1664 (2013).

6. Jiang, A., Schwartz, M., Bruck, J.: Error-correcting codes for rank modulation. In: Proceedings of IEEE International Symposium on Information Theory, pp. 1736-1740 (2008).

7. Jiang A., Schwartz M., Bruck J.: Rank modulation for flash memories. IEEE Trans. Inf. Theory 56(5), 2112-2120 (2010).

8. Kendall M., Gibbons J.D.: Rank Correlation Methods. Oxford University Press, New York (1990).

9. Kløve T., Lin T.-T., Tsai S.-C., Tzeng W.-G.: Permutation arrays under the Chebyshev distance. IEEE Trans. Inf. Theory 56(6), 2611-2617 (2010).

10. Kløve T.: Lower bounds on the size of spheres of permutations under the Chebychev distance. Des. Codes Cryptogr. 59, 183-191 (2011).

11. Ryser, H.J.: Combinatorial Mathematics. The Carus Mathematical Monographs \#14. The Mathematical Association of America, Buffalo (1963)

12. Sloane, N.J.A.: The On-Line Encyclopedia of Integer Sequences. http://www.research.att.com/ njas/ sequences/ 